\title{
COMPUTATION OF CLOSEST BIFURCATIONS IN POWER SYSTEMS
}

\author{
Fernando Alvarado \\ Ian Dobson \\ Member \\ Yi Hu \\ Fellow \\ Electrical \& Computer Engineering Department \\ Student Member \\ The University of Wisconsin-Madison
}

\begin{abstract}
Voltage collapse and blackout can occur in an electric power system when load powers vary so that the system loses stability in a saddle node bifurcation. This paper computes load powers at which bifurcation occurs and which are locally closest to given operating load powers. The distance in load power parameter space to this locally closest bifurcation is an index of voltage collapse and a minimum load power margin. The computations are illustrated for several power systems. Monte-Carlo optimization techniques are applied to obtain multiple minimum load power margins. The use of load power margin sensitivities to select system controls is discussed. Keywords: voltage collapse, saddle node bifurcation, load power margin, Monte-Carlo optimization.
\end{abstract}

\section{Introduction}

Consider the representation of active and reactive demands of an electric power system independently varying in a high dimensional load power parameter space. A given set of $m$ demands is represented by a point $\lambda_{0}$ in a parameter space of dimension $m$. Of importance is the set of critical loadings $\Sigma$ at which voltage collapse occurs because the operating equilibrium of the power system disappears in a saddle node bifurcation $[18,19]$. The set of critical loadings $\Sigma$ is composed of hypersurfaces in the parameter space (hypersurfaces are curved surfaces of dimension one less than the number of dimensions of the space). The dimension of the critical load hypersurface $\Sigma$ is $m-1$.

The 5 bus example power system from [4] has three loads, thus its parameter space is of dimension $m=6$. If we restrict our attention to the active powers, then $m=3$. Figure 1 illustrates the critical loading hypersurface $\Sigma$ for this system. An operating point $\lambda_{0}$ is also illustrated. To operate the power system securely with respect to voltage collapse, the distance from the operating point to $\Sigma$ must exceed some minimum threshold, otherwise corrective action should be taken to increase

93 SM 484-6 PWRS A paper recommended and approved by the IEEE Power System Engineering Committee of the IEEE Power Engineering Society for presentation at the IEEE/PES 1993 Summer Meeting, Vancouver, B.C., Canada, July 18-22, 1993. Manuscript submitted Jan. 4, 1993; made available for printing May 7, 1993.

PRINTED IN USA

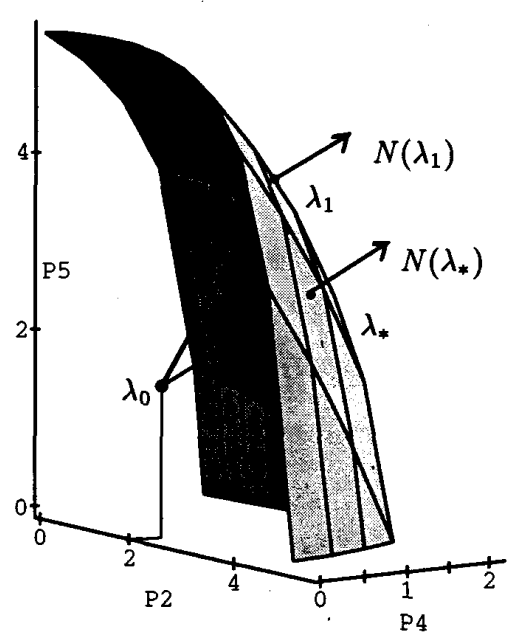

Fig. 1: Active load power parameter space for 9-load system indicating given direction and closest bifurcation load increase directions from a given load.

this distance. The point $\lambda_{*}$ on $\Sigma$ which is locally closest to $\lambda_{0}$ in a Euclidean sense is called a closest bifurcation and is illustrated. The line joining $\lambda_{0}$ to $\lambda_{*}$ is normal to $\Sigma$. The load increase from $\lambda_{0}$ to $\lambda_{*}$ is a worst case load increase and the closest distance $\left|\lambda_{*}-\lambda_{0}\right|$ is a power margin which measures the proximity of $\lambda_{0}$ to saddle node bifurcation and voltage collapse.

If a load forecast specifying the direction of load increase in load parameter space is available, a load power margin index $\ell$ assuming a direction of load increase can be computed $[4,9]$. The minimum load power margin index $\left|\lambda_{*}-\lambda_{0}\right|$ supplements the index $\ell$ to give a more complete description of the relation in parameter space of the base load powers $\lambda_{0}$ to the critical load powers $\Sigma$. If $\ell$ is sufficiently large but $\left|\lambda_{*}-\lambda_{0}\right|$ is small, a minor perturbation could precipitate voltage collapse. This paper computes a closest distance $\left|\lambda_{*}-\lambda_{0}\right|$ from $\lambda_{0}$ to $\Sigma$ for practical power systems using the iterative method from [14]. The method works by repeating the computation of $\ell$ for successive directions of load increase obtained from vectors normal to $\Sigma$.

We review previous work on closest bifurcations in power systems. Galiana and Jarjis [20] consider real power flow equations with constant voltage magnitudes, define a feasibility region in a real power injection parameter space, and present the idea of computing a 
closest instability in this parameter space. Using a conjecture that the feasibility region boundary $\Sigma$ is convex, Galiana and Jarjis parameterize $\Sigma$ and define a real power margin $D$ which is the perpendicular distance from the operating real power injections $\lambda_{0}$ to the tangent hyperplane of $\Sigma$ with normal $N$. Minimizing $D$ with conjugate gradient methods yields a minimum real power margin. This computation is illustrated using a 6 bus system. In a subsequent paper [22] they define a feasibility region in real and reactive power injection parameter space augmented with the voltage magnitudes of PV buses. A non-Euclidean minimum parameter space margin is defined and computed using constrained Fletcher-Powell minimization.

Jung et al. [24] suggest a gradient projection optimization method to compute a minimum load power margin $\left|\lambda_{*}-\lambda_{0}\right|$. Sekine et al. [26] approximate a minimum load power margin by gradient descent on the determinant of the Jacobian.

Kumano et al. [28] suggest computing $\left|\lambda_{*}-\lambda_{0}\right|$ by optimization methods in state space. Let $S$ be the set of critical equilibria in state space at which the Jacobian is singular. The necessary condition for the critical equilibrium corresponding to $\lambda_{*}$ is that the gradient of $\left|\lambda_{*}-\lambda_{0}\right|^{2}$ with respect to the state vector $x$ is parallel to the normal vector to $S$. The correct formula for the normal vector to $S$ in state space is $w f_{x x} v$, where $w$ and $v$ are the left and right eigenvectors corresponding to the zero eigenvalue of the Jacobian $f_{x}$ and $f_{x x}$ is the Hessian of $f$. Reference [28] uses $v$ as the normal vector of $f$. This appears to be valid only for the case of a single line and infinite bus in rectangular coordinates.

The use of $\left|\lambda_{*}-\lambda_{0}\right|$ and other indices in determining the costs of secure power system operation is explained in Alvarado et al. [3].

Dobson et al. propose a direct method to compute $\left|\lambda_{*}-\lambda_{0}\right|$ in $[11,14]$. Dobson and Lu propose an iterative method to compute $\left|\lambda_{*}-\lambda_{0}\right|$ in [14] and $[10,13]$. However, [14] tests the method only in a 5 bus system.

The main objective of this paper is to demonstrate the iterative method on larger systems, to present some numerical experiments, and to provide additional insight into the method. Practical computation of closest bifurcations requires sparse matrix techniques [2].

\section{Preliminaries}

The iterative method to compute a closest saddle node bifurcation has two components: a formula for the normal vector to $\Sigma$ and a method for finding the load power margin $\ell$ assuming a direction of load increase.

The static model for the power system has the form:

$$
0=f(x, \lambda)
$$

where $x$ is a state vector which includes bus voltage phasors, and $\lambda$ is a vector of active (and possibly also reactive) power demands of dimension $m$. Loads representing fixed injections or zero-injection nodes should be omitted from $\lambda$, as well as any other loads not of direct interest. This formulation represents fixed injection or zero injection nodes accurately. Intelligent selection of a few representative loads (as elements of $\lambda$ ) is useful in many studies. Underlying differential equation models are assumed but are not necessary for computations $[12,14]$. At a saddle node bifurcation specified by load powers $\lambda_{1} \in \Sigma$ the corresponding operating equilibrium $x_{1}$ is degenerate and the Jacobian $\left.f_{x}\right|_{\left(x_{1}, \lambda_{1}\right)}$ has a zero eigenvalue with corresponding left eigenvector $w_{1}$ of dimension $n$, the number of states. Under standard generic assumptions, $\Sigma$ is a smooth hypersurface near $\lambda_{1}$ and a normal vector to the hypersurface at $\lambda_{1}$ is $[8,12]$

$$
N\left(\lambda_{1}\right)=w_{1} f_{\lambda}
$$

where $f_{\lambda}$ is the Jacobian of $f$ with respect to $\lambda\left(f_{\lambda}\right.$ is an $n \times m$ matrix). For the case where $\lambda$ corresponds to demands and these appear as unscaled linear entries in $f$, the product $w_{1} f_{\lambda}$ amounts to the selection of certain entries of $w_{1}$ and the discarding of the rest. The vector $w_{1}$ is written as a row vector and its sign is chosen so that an increase of load in the direction $N\left(\lambda_{1}\right)$ leads to disappearance of the operating equilibrium.

Suppose we specify a particular direction of future load increase from a demand $\lambda_{0}$. That is, we specify a ray in parameter space based at $\lambda_{0}$ with a unit vector $n_{0}$ so that the load powers $\lambda$ along the ray are given by

$$
\lambda=\lambda_{0}+\ell^{\prime} n_{0}
$$

as the loading factor $\ell^{\prime}$ assumes positive real values. We can compute the closest saddle node bifurcation along this ray of load increase (and the corresponding critical loading factor $\ell$ ) by requiring that $\lambda_{1}=\lambda_{0}+\ell n_{0} \in \Sigma$. Since $n_{0}$ is a unit vector, $\ell=\left|\lambda_{1}-\lambda_{0}\right|$. $\ell$ is a voltage collapse index measuring the load power margin assuming the direction of load increase. This computation is done either by continuation methods $[21,7,1]$ or by direct or optimization methods $[4,24,9]$. Seydel [27] gives an entry to the extensive numerical analysis literature on both continuation and direct methods.

For this paper we use a variant of the direct method $[15,5,9]$ in which solution of the following equations by Newton's method yields the bifurcating equilibrium $x_{1}$, load power margin $\ell$ and left eigenvector $w_{1}$ :

$$
\begin{aligned}
f\left(x_{1}, \lambda_{0}+\ell n_{0}\right) & =0 \\
\left.w_{1} f_{x}\right|_{\left(x_{1}, \lambda_{0}+\ell n_{0}\right)} & =0 \\
w_{1} c-1 & =0
\end{aligned}
$$

Equation (4) states that $x_{1}$ is an equilibrium at parameter $\lambda_{1}=\lambda_{0}+\ell n_{0}$ and equation (5) states that the Jacobian $f_{x}$ evaluated at $\left(x_{1}, \lambda_{1}\right)$ is singular with left eigenvector $w_{1} . \quad c \in \mathbf{R}^{p}$ is a fixed vector and (6) ensures that the left eigenvector $w_{1}$ is nonzero. Equations (4)-(6) use a left eigenvector $w_{1}$ in place of the right eigenvector used in some earlier formulations. The left eigenvector formulation is preferred over the right eigenvector formulation due to its more direct interpretation in terms of a direction normal to the boundary.

The use of Newton's method for the direct solution of these equations requires the simultaneous solution of equations (4)-(6). One computational difficulty with this approach is that the dimension of the Jacobian matrix required to solve this problem is about twice the dimension of $f_{x}$, although it remains very sparse. The convergence of the direct method is excellent provided a sufficiently close initial guess is provided. References $[5,6]$ give guidelines for the choice of initial guesses. 
As an alternative to direct methods, it is possible to use continuation methods to determine the bifurcation point $x_{1}$. In this approach, the bifurcation point $x_{1}$ is determined from equation (4) alone, augmented by a continuation variable parameter. Continuation methods for power flow applications are reported in a number of papers, including $[1,5,7]$. Other techniques of avoiding singularities in the vicinity of bifurcation points include those of [23]. When the Jacobian $f_{x}$ becomes singular during the continuation process, this is an indication of having attained $\Sigma$. At this point, equations (5) and (6) can be solved simultaneously to obtain $\omega_{1}$. This method never requires a matrix larger than $n+1$ and is non-iterative.

Our software implements both the direct and the continuation method $[5,6]$. The continuation method followed by the subsequent computation of $\omega_{1}$ is the recommended implementation variant for larger problems. However, in many of the tests that follow it was convenient to use the direct method because in our experiments we were interested more in high accuracy than in speed. Although no exact timings were obtained, our evidence from numerous experiments suggests that the determination of the boundary in a given direction was on the average comparable to about 10 power flows for systems of up to about 2000 buses, slightly more for the direct method. With the direct method there appeared to be significant degradation in performance relative to a single power flow for larger system sizes. This behavior was not apparent for the continuation based method, thus our recommendation.

Reactive power and other limits in the power flow equations often result in added complexity and increased iteration counts in most methods. The normal vectors at the boundary are those that are applicable to the situation with the limits active at the given solution point. In some cases encountering limits can create an immediate instability before the saddle node bifurcation is reached [16] and the load power margin computation is misleading. We neglect this phenomenon, but Cañizares [5] observed a range of practical cases in which the error introduced by neglecting this phenomenon was very small.

\section{Iterative Method}

The load power margin $\ell$ and the computation of the normal vector of $\Sigma$ may be iterated to compute the direction $n_{*}$ and parameter value $\lambda_{*}$ of a locally closest saddle node bifurcation and hence the minimum power margin $\left|\lambda_{*}-\lambda_{0}\right|$. The procedure is as follows [14]:

(0) Let $n_{0}$ be an initial guess for the direction $n_{*}$.

(1) Given $n_{i-1}$, compute the saddle node bifurcation along the ray given by $n_{i-1}$; that is, compute $\ell_{i}$, $\lambda_{i}, x_{i}$ so that $\lambda_{i}=\lambda_{0}+n_{i-1} \ell_{i} \in \Sigma$.

(2) Compute the left eigenvector $w_{i}$ of $\left.f_{x}\right|_{\left(x_{i}, \lambda_{i}\right)}$ corresponding to the zero eigenvalue.

(3) Set $n_{i}=N\left(\lambda_{i}\right)=w_{i} f_{\lambda}$.

(4) Iterate steps $1,2,3$ until convergence within a tolerance of $n_{i}$ to a value $n_{*}$. Then $\lambda_{*}=\lambda_{0}+\ell_{*} n_{*}$.
The computationally critical step is (1), which is solved by the methods outlined in the previous section. The direction $n_{*}$ of a locally closest bifurcation is parallel to the normal vector $N\left(\lambda_{*}\right)$ of $\Sigma$ at $\lambda_{*}$, and $n_{*}$ is a fixed point of the iteration. The iteration converges in one step in the hypothetical case of $\Sigma$ being a hyperplane.

The iteration minimizes $\left|\lambda_{*}-\lambda_{0}\right|$ on a series of tangent hyperplane approximations to $\Sigma$. At each iteration, $n_{i}=N\left(\lambda_{i}\right)$ indicates the direction of the point closest to $\lambda_{0}$ on the tangent hyperplane $T \Sigma_{\lambda_{i}}$ to $\Sigma$ at $\lambda_{i}$. Supporting theory for the iterative method is presented in $[10,13]$.

A direct method for computing a closest bifurcation $[11,14]$ is not considered in this paper because it requires the curvature of $\Sigma$ at $\lambda_{*}$. The curvature is difficult to compute directly $[10,14]$. An important advantage of the iterative method is that its convergence to a solution ensures that the solution is a locally closest bifurcation and no check on the curvature is required.

\section{Computational Results and Discussion}

The computational methods described above are applied to several power systems ranging from 3 to 173 buses. The iterates $\left|\lambda_{i}-\lambda_{0}\right|$ and the final minimum load power margins $\left|\lambda_{*}-\lambda_{0}\right|$ are summarized in Table 1. A "reasonable" initial loading direction vector $n_{0}$ for the iterative method was arbitrarily chosen by assigning unity to all generators and real power loads, and 0.2 to all reactive power loads. An exception is the 173-bus system for which a demand growth direction was available.

Cañizares [5] established the computational performance of our software for the determination of a bifurcation point along a given ray. Solution times were roughly equivalent to 10 power flows each. Since the iterative method of this paper requires the bifurcation point along a given ray to be determined between 3 to 15 times, the total solution time for the iterative method to compute the minimum load power margin is roughly equivalent to 100 power flows.

Based on these 6 sets of experiments, it is observed that few iterations (sometimes only two or three) are sufficient to converge to the closest bifurcation. This suggests that the portions of $\Sigma$ encountered during the iterations are relatively flat.

The implications of knowing minimum load power margins are significant. These margins represent the true worse case load increases for system loadability with respect to voltage collapse (see, however, the discussion on multiple minima in the next section). While in some cases it is obvious after the fact what loading patterns are critical, in other cases these are not obvious a priori. In some of the cases tested, the direction of load increase to a closest bifurcation corresponds to an increase of loads throughout the system. However, in other cases the direction of load increase corresponds to the loading of primarily one or two buses. These studies give, in precise terms, the worse loadabilities and margins and can therefore serve as useful indicators for security dispatch and other means to monitor and enhance system security. This type of information 
Table 1: Minimum load power margins

\begin{tabular}{|c|l|l|}
\hline System & iteration & $\begin{array}{l}\text { margins } \\
\left|\lambda_{i}-\lambda_{0}\right|\end{array}$ \\
\hline 3-bus & 0 & 588.2 \\
& 1 & 371.6 \\
& 2 & 356.7 \\
& 3 & 355.5 \\
& $*$ & 355.4 \\
\hline 17-bus & 0 & 671.1 \\
& 1 & 283.0 \\
& 2 & 268.7 \\
& 3 & 262.3 \\
& 4 & 258.6 \\
& $*$ & 253.4 \\
\hline 24-bus & 0 & 978.5 \\
Reliability & 1 & 470.7 \\
& 2 & 442.2 \\
& 3 & 398.5 \\
& 4 & 380.9 \\
& $*$ & 378.3 \\
\hline 39-bus & 0 & 1783.8 \\
New England & 1 & 1163.9 \\
& 2 & 1008.2 \\
& 3 & 734.9 \\
& 4 & 716.0 \\
& $*$ & 714.8 \\
\hline 173-bus & 0 & 474.4 \\
Area load & 1 & 274.3 \\
& $*$ & 274.3 \\
\hline Entire load & 0 & 2409.9 \\
& 2 & 268.2 \\
& $*$ & 267.5 \\
& & 267.5 \\
\hline
\end{tabular}

is particularly valuable in unusual operating regimes or in planning when the pattern of load increase is not known.

\section{Load power margin sensitivities}

The sensitivities of load power margins to power system controls can be used to select system controls that best increase the load power margin. Once the system controls have been selected, the approximate amount of control required to achieve the required increase in load power margin can be computed. The first order sensitivities of the worst case load power margin $\left|\lambda_{*}-\lambda_{0}\right|$ and the given direction load power margin $\ell=\left|\lambda_{1}-\lambda_{0}\right|$ are both easy to compute $[13,14,15]$. Let $c$ be a vector of any power system controls or parameters and let $p=(\lambda, c)$ be an augmented vector containing both load powers and the parameters $c$. Then according to [15] the sensitivity of $\left|\lambda_{*}-\lambda_{0}\right|$ with respect to $p$ is

$$
\left|\lambda_{*}-\lambda_{0}\right|_{p}=\frac{-w f_{p}}{w f_{p} \cdot n_{*}}
$$

and the sensitivity of $\ell$ with respect to $p$ is

$$
\ell_{p}=\frac{-w f_{p}}{w f_{p} \cdot n_{0}}
$$

In both (7) and (8), $f_{p}$ is the Jacobian of $f$ with respect to the vector $p$ and $w$ is the left eigenvector corresponding to the zero eigenvalue of the Jacobian $f_{x}$ evaluated at the bifurcation. The vector $n_{*}=\left(\lambda_{*}-\right.$ $\left.\lambda_{0}\right) /\left|\lambda_{*}-\lambda_{0}\right|$ is the worst case direction of load increase. $w f_{p}$ can be interpreted as a normal to the hypersurface in the loading and parameter space of critical $p$ yielding bifurcation. $f_{p}$ is usually easy to calculate.

We discuss the use of the sensitivity formula (7) to control $\left|\lambda_{*}-\lambda_{0}\right|$. Equation (8) may similarly be used to adjust the index $\ell$ as illustrated below. If the worst case load power margin $\left|\lambda_{*}-\lambda_{0}\right|$ is too small, less than a preselected safety margin $L$, we may seek to control the system to restore $\left|\lambda_{*}-\lambda_{0}\right|$ to at least $L$. If the left eigenvector $w$ is computed at the bifurcation corresponding to $\lambda_{*}$, then the sensitivity $\left|\lambda_{*}-\lambda_{0}\right|_{p}$ can be computed with (7) where the vector $p$ includes all available power system controls. (Adding extra controls to $p$ affects only $f_{p}$ and is computationally cheap.) Then a subset of controls which have relatively large components in $\left|\lambda_{*}-\lambda_{0}\right|_{p}$ and are appropriate to the severity of the risk of voltage collapse should be selected. For example, load shedding controls could be avoided unless indexes show that voltage collapse is probable. Once the choice and relative proportion of controls is selected, then the amount of control action to restore the system to the safety margin can be computed from (7).

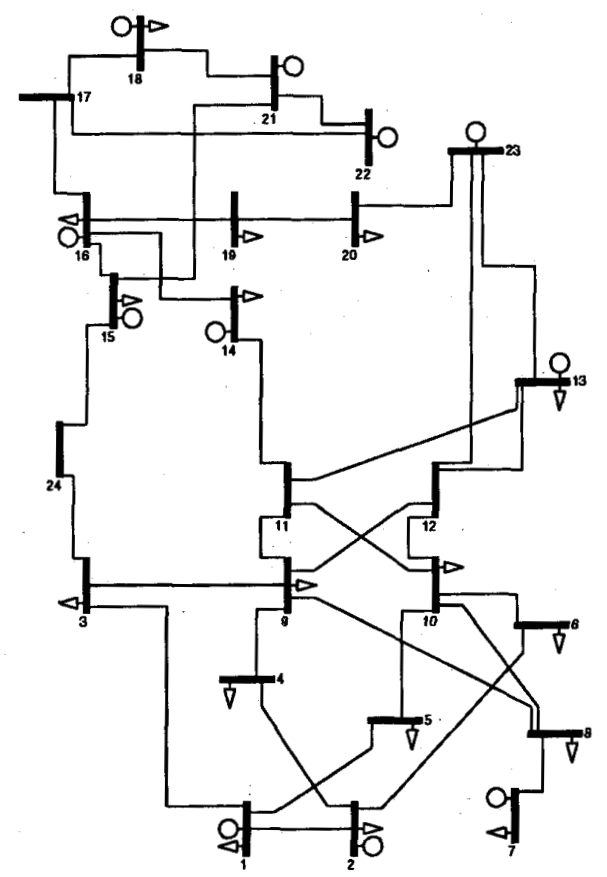

Fig. 2: One-line diagram for the 24-bus system

As a specific example of the usefulness of sensitivity calculations, we predict the effect on the given-direction voltage collapse margin $\ell$ of a decrease in demand at bus 4 for the 24 bus system illustrated in figure 2. To make the example more interesting, we first obtained a new operating point close to the boundary by moving along the given direction of demand increase. The sensitivity 
factor for bus 4 at this point is $\ell_{p 4}=0.4443$. The givendirection distance is $53.5 \mathrm{MW}$ with no load shedding. $A$ decrease in load at bus 4 increases the margin by a predicted amount:

$$
\left|\lambda_{*}-\lambda_{0}\right|^{\text {new }}=\left|\lambda_{*}-\lambda_{0}\right|^{\text {old }}+\ell_{p 4} \Delta \lambda_{p_{4}}
$$

Table 2 illustrates the predicted and calculated new distances for different amounts of shed load ranging from $1 \mathrm{MW}$ to $59 \mathrm{MW}$ (the total active load at the bus). The sensitivity prediction is excellent for small perturbations and adequate for full load shedding. We conclude for this case that attaining a given security margin $L$ using the sensitivity formulas to adjust the reactive injection or active power demand is a simple matter.

Table 2: Effect of load shedding on load power margin $\ell$ (computed versus sensitivity-predicted calculations).

\begin{tabular}{|c|c|c|}
\hline Load shed (MW) & Computed & Predicted \\
\hline 0 & 53.5 & 53.5 \\
1 & 53.9 & 53.9 \\
5 & 55.4 & 55.7 \\
10 & 57.3 & 57.9 \\
59 & 73.3 & 79.6 \\
\hline
\end{tabular}

\section{Finding Multiple Closest Bifurcations}

When the iterative method converges, it converges to a locally closest bifurcation $\lambda_{*}$ which is not necessarily the globally closest bifurcation. Multiple locally clósest bifurcations may exist and hence multiple minima of the load power margin. While the closest bifurcation in a direction of "reasonable" demand increase is probably most important from a practical point of view, it is also of interest to investigate other closest bifurcations. In particular, a heavily stressed system might be close to several hypersurfaces of $\Sigma$ and monitoring and corrective action might need to take into account a minimum load power margin to each of the hypersurfaces. Reference [14] gives a simple power system example with multiple hypersurfaces corresponding to voltage collapse in decoupled areas of the power system. Limits and other types of system discontinuities might also produce multiple closest bifurcation points.

It is possible for a single hypersurface to have multiple locally closest bifurcations if the hypersurface were "corrugated" and this could limit the usefulness of the closest bifurcation computation. However, a conjecture of [22] implies that $\Sigma$ is convex and convexity precludes corrugation. We have not detected any non-convex portions of $\Sigma$ in our computations to date. (Moderate or small amounts of non-convexity of $\Sigma$ would produce oscillatory convergence to $\lambda_{*}$.) However, proving or disproving the convexity of $\Sigma$ for, say, the load flow equations remains an open problem.

We compute multiple minimum load power margins using Monte Carlo optimization [25]. Initial directions $n_{0}$ for the iterative method are randomly generated by choosing vectors from a uniform distribution on an $m$ dimensional hypercube corresponding to the $m$ distinct demands. Both active and reactive power are treated as independent variables. The iterative method is run for

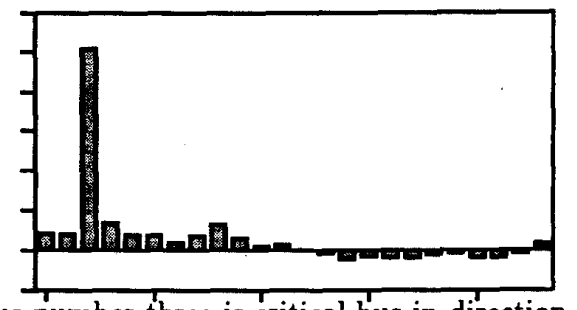

(a) Bus number three is critical bus in direction 1 .

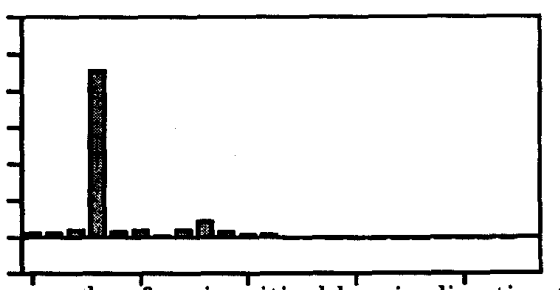

(b) Bus number four is critical bus in direction 2.

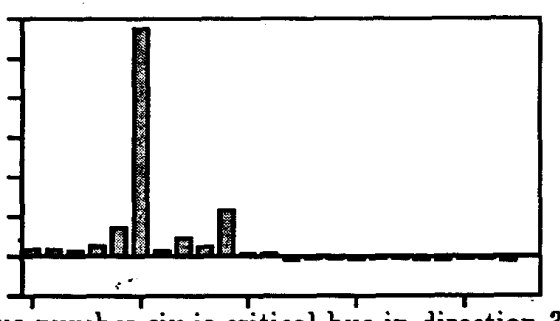

(c) Bus number six is critical bus in direction 3 .

Fig. 3: Normal to critical loading hypersurface at three distinct closest bifurcation points for the 24 bus system. Active power components in arbitrary units along each vertical axis, buses from 1 to 24 along the horizontal axes.

each of these initial load increase directions. All distinct closest bifurcations and their corresponding minimum load power margins are shown in Table 3 . Boldface numbers correspond to the distances obtained using the pre-specified "reasonable" initial direction vector. 150 initial directions are used for the 173 bus systems and larger numbers of initial directions are used for the smaller systems. Only one closest bifurcation is found for the 3-bus, 17-bus and 173-bus systems, three distances are identified for the 24-bus system, and 4 distances are found for the 39-bus system.

For the 24-bus system one of the directions (italicized) results in a shorter distance to the boundary than the initial direction provided. Figure 3 illustrates the active power component of the three distinct directions identified for the 24 bus system. Since the eigenvectors can be scaled arbitrarily, the vertical axis is in arbitrary units, indicating only the relative importance of each location. Table 4 illustrates in detail the active and reactive base case load, the load at the givendirection boundary, and the three closest bifurcation loads. From this table (and from the figures) it is seen that collapse is easiest to attain by either increases pri- 
Table 3: Multiple minimum load power margins

\begin{tabular}{|r|c|c|}
\hline System & $m$ & Margin (MW) \\
\hline 3-bus & 4 & $\mathbf{3 5 5}$ \\
\hline 17-bus & 14 & $\mathbf{2 5 3}$ \\
\hline 24-bus & 34 & $\mathbf{3 7 8}$ \\
& & 322 \\
& & 389 \\
\hline 39-bus & 38 & $\mathbf{7 1 5}$ \\
& & 887 \\
& & 1074 \\
& & 1103 \\
\hline 173-bus (area) & 56 & $\mathbf{2 7 4}$ \\
\hline 173-bus (total) & 148 & $\mathbf{2 6 7}$ \\
\hline
\end{tabular}

marily in the reactive and active powers at bus 4 , or by increases primarily at bus 3 , or by increases primarily at bus 6 . These three buses are thus identified as the critical buses from the perspective of voltage collapse. The minimum distance(s) to the boundary (and consequently the security of the system) can be improved by increasing reactive power support at these buses, or by reducing active power demand, or both.

There are situations (not illustrated due to space limitations) where a number of buses all having more or less the same effect on the minimum distance are found. In these cases, the minimum distance algorithm identifies weak areas in the system. The minimum distance algorithm can be used to study both active and reactive power variations, or it can be used for reactive powers alone or for active powers alone. Depending on the application, these different types of studies provide different insights into the needs of a specific system. Only further experimentation with these variants on larger systems will establish which variant is most appropriate to a specific system study.

This paper has not considered the efficient implementation of globally minimum load power margins. We expect this computation to be difficult because recent work by Coxson [17] strongly suggests that the global minimum problem is NP-hard.

\section{Conclusions}

The ability to determine closest bifurcations and minimum load power margins for large systems is important from the perspective of security and remedial actions after contingencies. The minimum load power margin is a useful index of proximity to voltage collapse. This margin does not require an assumption of the pattern of load increase, but if the pattern of load increase is available, then the minimum load power margin complements the given-direction load power margin. This paper demonstrates the feasibility of practical computation of closest saddle node bifurcations and the associated minimum load power margins. The iterative method outlined in this paper requires only repeated load power margin computations and use of the Jacobian at the bifurcation. The paper also illustrates the practical use of margin sensitivity formulas.

The security boundary $\Sigma$ defines a fundamental limit on power system performance and must be avoided to
Table 4: Comparison of demands for three minimum distance directions, 24 bus system

\begin{tabular}{|r|r|r|r|r|r|r|r|r|r|r|}
\hline & \multicolumn{2}{|c|}{$\begin{array}{c}\text { Base } \\
\text { load } \lambda_{0}\end{array}$} & \multicolumn{2}{c|}{$\begin{array}{l}\text { Load } \lambda_{1} \\
\text { given }\end{array} n_{0}$} & \multicolumn{5}{|c|}{ Closest load $\lambda_{*}$} \\
\hline Bus & $\mathrm{P}$ & $\mathrm{Q}$ & $\mathrm{P}$ & $\mathrm{Q}$ & $\mathrm{P}$ & $\mathrm{Q}$ & $\mathrm{P}$ & $\mathrm{Q}$ & $\mathrm{P}$ & $\mathrm{Q}$ \\
\hline 1 & 108 & 22 & 340 & 68 & 111 & 22 & 122 & 22 & 114 & 22 \\
2 & 97 & 20 & 329 & 66 & 100 & 20 & 110 & 19 & 103 & 20 \\
3 & 180 & 37 & 412 & 83 & 186 & 57 & 347 & 365 & 184 & 46 \\
4 & 74 & 15 & 306 & 61 & 205 & 302 & 96 & 47 & 83 & 26 \\
5 & 71 & 14 & 303 & 60 & 75 & 18 & 84 & 19 & 94 & 64 \\
6 & 136 & 28 & 368 & 74 & 142 & 34 & 149 & 36 & 317 & 344 \\
7 & 125 & 25 & 357 & 71 & 126 & 25 & 131 & 24 & 130 & 25 \\
8 & 171 & 35 & 403 & 81 & 176 & 46 & 183 & 49 & 186 & 62 \\
9 & 175 & 36 & 407 & 82 & 187 & 86 & 196 & 93 & 183 & 57 \\
10 & 195 & 40 & 427 & 86 & 200 & 48 & 205 & 50 & 232 & 150 \\
13 & 265 & 54 & 497 & 100 & 264 & 54 & 265 & 54 & 262 & 54 \\
14 & 194 & 39 & 426 & 85 & 193 & 39 & 191 & 39 & 192 & 39 \\
15 & 317 & 64 & 549 & 110 & 316 & 64 & 309 & 63 & 315 & 64 \\
16 & 100 & 20 & 332 & 66 & 99 & 20 & 94 & 20 & 98 & 20 \\
18 & 333 & 68 & 565 & 114 & 332 & 68 & 326 & 68 & 331 & 68 \\
19 & 181 & 37 & 413 & 83 & 180 & 36 & 176 & 37 & 178 & 37 \\
20 & 128 & 26 & 360 & 72 & 127 & 26 & 124 & 25 & 125 & 26 \\
\hline & 2850 & & 6794 & & 3019 & & 3108 & & 3127 & \\
\hline
\end{tabular}

prevent voltage collapse. Despite the importance of $\Sigma$, little is established about its geometry in practice, simulation or theory. This paper contributes to the understanding of the geometry of the set of critical loads by computing minimum load power margins and addressing the issue of multiple minimum load power margins.

\section{Acknowledgements}

Support in part from NSF grants ECS-9157192, ECS8907391 and EPRI contracts RP 8010-30, RP 400014 is gratefully acknowledged.

\section{REFERENCES}

[1] V. Ajjarapu, C. Christy, "The Continuation Power Flow: A Tool for Steady State Voltage Stability Analysis," IEEE PICA, May 1991, Baltimore, MD

[2] F. L. Alvarado, W. F. Tinney, and M. K. Enns. Sparsity in large-scale network computation. In C. T. Leondes, editor, Advances in Electric Power and Energy Conversion System Dynamics and Control, volume 41 of Control and Dynamic Systems, pages 207-272. Academic Press, 1991. Part 1.

[3] F.L. Alvarado, Y. Hu, D. Ray, R. Stevenson and E. Cashman, "Engineering Foundations for the Determination of Security Costs," IEEE Trans. on Power Systems, Vol. 6, Aug. 1991, pp. 1175-1182.

[4] F.L. Alvarado and T.H. Jung, "Direct Detection of Voltage Collapse Condition," in [18], pp 5.23-5.38.

[5] C.A. Cañizares, F.L. Alvarado, "Computational experience with the point of collapse method on very large $\mathrm{AC} / \mathrm{DC}$ systems," in [19], pp. 103-111.

[6] C.A. Cañizares, F.L. Alvarado, "Point of collapse and continuation methods for large AC/DC systems," 92 WM 103-2-PWRS IEEE/PES Winter Meeting, New York, Jan. 1992. 
[7] H.D. Chiang, W. Ma, R.J. Thomas, J.S. Thorp, “A tool for analyzing voltage collapse in electric power systems" Proc. of PSCC, Graz, Austria, Aug. 1990.

[8] S.N. Chow, J. Hale, Methods of bifurcation theory, Section 6.2, Springer-Verlag, NY, 1982.

[9] T. Van Cutsem, "A method to compute reactive power margins with respect to voltage collapse", IEEE Trans. on Power Systems, Vol. 6, Feb. 1991, pp. 145-156.

[10] I. Dobson, "Computing a closest bifurcation instability in multidimensional parameter space", report ECE-914, ECE Dept., U. of Wisconsin, Madison, WI, Apr. 1991, revised Feb. 1992. To appear in Journal of Nonlinear Science.

[11] I. Dobson, L. Lu and Y. Hu, "A Direct Method for Computing the Closest Saddle Node Bifurcation in the Load Power Parameter Space of an Electric Power System," IEEE International Symposium on Circuit and System, Singapore, June 1991, pp. 3019-3022.

[12] I. Dobson, "Observations on the Geometry of Saddle Node Bifurcations and Voltage Collapse in Electric Power Systems," IEEE Trans. on Circuits and Systems, Part 1, Vol. 39, No. 3, March 1992, pp. 240-243.

[13] I. Dobson, "An iterative method to compute the closest saddle node or Hopf bifurcation in multidimensional parameter space", Proceedings of the IEEE International Symposium on Circuits and Systems, San Diego, CA, May 1992, pp. 2513-2516.

[14] I. Dobson, L. Lu, "New methods for computing a closest saddle node bifurcation and worst case load power margin for voltage collapse," 92 SM 587-6 PWRS, IEEE PES Summer meeting, Seattle, WA, July 1992.

[15] I. Dobson, L. Lu, "Computing an optimum direction in control space to avoid saddle node bifurcation and voltage collapse in electric power systems", in [19] and IEEE Transactions on Automatic Control, Vol 37, No. 10, October 1992, pp. 1616-1620.

[16] I. Dobson, L. Lu, "Voltage collapse precipitated by the immediate change in stability when generator reactive power limits are encountered," IEEE Transactions on Circuits and Systems, Part 1, Vol 39, No. 9, September 1992, pages 762-766.

[17] G. Coxson, "Computational Complexity of Robust Stability and Regularity in Families of Linear Systems," $\mathrm{PhD}$ thesis, University of Wisconsin-Madison, April 1993.

[18] L.H. Fink, ed., Proceedings: Bulk power system voltage phenomena voltage stability and security, EPRI Report EL-6183, Potosi, Missouri, Jan. 1989.

[19] L.H. Fink, ed., Proceedings: Bulk power system voltage phenomena, voltage stability and security ECC/NSF workshop, Deep Creek Lake, MD, Aug. 1991, ECC Inc., 4400 Fair Lakes Court, Fairfax, VA 22033-3899.

[20] F.D. Galiana, J. Jarjis, "Feasibility constraints in power systems", A 78 560-5 IEEE PES Summer meeting, Los Angeles, CA, July 1978.

[21] K. Iba, H. Suzuki, M. Egawa, T. Watanabe, "Calculation of critical loading condition with nose curve using homotopy continuation method", IEEE Trans. on Power Systems, Vol. 6, May 1991, pp. 584-593.

[22] J. Jarjis, F.D. Galiana, "Quantitative analysis of steady state stability in power networks", IEEE Trans. on Power Apparatus and Systems, Vol. PAS-100, Jan. 1981, pp. 318-326.
[23] R. Jean-Jumeau and H.D. Chiang. Parameterization of the load-flow equations for eliminating ill-conditioning in the neighborhood of singular points. 92 SM 5868-PWRS IEEE/PES Summer Meeting, Seattle, WA, July 1992.

[24] T.H. Jung, K.J. Kim, F.L. Alvarado, "A marginal analysis of the voltage stability with load variations", Proc. of PSCC, Graz, Austria, August 1990, pp. 1196-1201.

[25] R.Y. Rubinstein, Monte Carlo Optimization, Simulation and Sensitivity of Queueing Networks, John Wiley \& Sons, Inc., 1986.

[26] Y. Sekine, A. Yokoyama, T. Kumano, "A method for detecting a critical state of voltage collapse", in [18].

[27] R. Seydel, "From Equilibrium to Chaos," Elsevier Science Publishing Co., NY, 1988.

[28] T. Kumano, H. Tanaka, A. Yokoyama, Y. Sekine, Detection of closest critical point and suboptimal preventive control of voltage stability, Electrical engineering in Japan, Vol. 111, No. 3, 1991, pp. 89-98.

Fernando Alvarado (F'93) obtained a a Ph.D. from the University of Michigan in 1972 . He is currently a Professor at the University of Wisconsin in Madison in the Department of Electrical and Computer Engineering. His main areas of interest are computer applications to power systems and large sparse matrix problems.

Ian Dobson (M'89) received the BA in Mathematics from Cambridge, England in 1978 and joined the University of Wisconsin-Madison faculty after receiving the $\mathrm{PhD}$ in Electrical Engineering from Cornell in 1989. His industrial experience working in Britain from 1978 to 1983 included writing a general simulation of switching power supplies. His current interests include voltage collapse and other power system instabilities and applications of bifurcation theory, nonlinear dynamics and chaos.

Yi Hu (S'90) received a BSEE degree from the Beijing Polytechnic University in 1982 and a Ph.D. degree from the University of Wisconsin-Madison in 1992. His interests include power electronics, power system security and reliability, voltage collapse and load forecasting using neural networks. 


\section{Discussion}

M. A. Pai (University of Illinois, Urbana-Champaign): This is a well written and practically oriented paper with good theoretical support. The authors have moved away from the traditional P-V curve and have made computations directly in the parameter space. Given an operating point $\lambda_{0}$ in the parameter space, it computes a point on the surface $\Sigma$ closest to $\lambda_{0}$. In some sense it is a worse case scenario for voltage collapse. $\Sigma$ is the bifurcation boundary. The point $\lambda_{0}$ is structurally stable, i.e., for minor perturbations in the parameters, the new operating point remains close to $\lambda_{0}$. Thus points on $\Sigma$ also represent the structural stability limit (SSL) which is also the bifurcation boundary [A]. As the authors point out there are many ways to approach $\Sigma$ from $\lambda_{0}$ and they have provided a measure of the minimum distance. The authors' comments are requested for the following.

1. Is the algorithm flexible to include tie line powers in $\lambda$ or will they need some distribution factors?

2. Given the flexibility of the algorithm, how best they propose to present the results to the operator in real time?

3. Will the inclusion of system and or load dynamics shrink the SSL?

\section{Reference}

[A] Practical Numerical Algorithms For Chaotic Systems, T. S. Parker and L. O. Chua, Springer-Verlag, New York, 1989. Manuscript received June 30, 1993.

M. K. Pal: This paper describes a method to compute the load power margin to a locally closest bifurcation point. In addition to the direction of load increase $\left(n_{*}\right)$ which the authors compute, the direction of the change in generation to meet that load increase will also determine the minimum load power margin. There could be a number of valid options for increasing the generation to match the increased load and losses. Have the authors used any specific scheme, or looked for the worst case generation increase? The question of selecting the generation pattern needs to be settled before the computed minimum power margins can be said to represent the "true worst case load increases for system loadability."

The purpose of computing a minimum load power margin is not quite clear to us. The authors say that if $\left|\lambda_{*}-\lambda\right|$ is small, a minor perturbation could precipitate voltage collapse. This is not true - it all depends on load characteristics. If in a specific situation (dictated by load characteristics) it is not desirable to operate close to a bifurcation point $\left(\left|\lambda_{*}-\lambda\right|\right.$ small), sensitivity information (or, the eigenvalues) obtained from the power flow Jacobian may be a better guide to use.

In most situations, however, it is not necessary to operate a system with a generous "worst case" load power margin, since the load increase may not follow that direction as indicated by operating experience. In the unlikely event of the worst case load increase some sort of emergency measures may be taken. This will avoid the economic penalty of allowing for margins that may hardly be justified except in certain specific situations. As an example, consider the results shown in Table 2 of the paper. In order to increase the load power margin for the system by 20 MW, $60 \mathrm{MW}$ of load is needed to be shed at bus 4 of the system of Figure 2 of the paper.

Our main concern is, however, the basic premise of the paper that "voltage collapse occurs in an electric power system when load powers vary so that the system loses stability in a saddle node bifurcation." It has been clearly demonstrated that, in general, a saddle node bifurcation has nothing to do with voltage instability and collapse [A]. It merely represents a network limit for a given load-generation distribution. We recognize that this limit has important implications in system planning. Voltage instability is, however, determined by load characteristics and the type of voltage control in effect at the time of the instability [B-C]. Certain load characteristics - voltage control combinations can precipitate voltage collapse well before a saddle node bifurcation. On the other hand, for many different load types, one can continue loading the system after the bifurcation point is reached, without any danger of voltage instability and collapse, although the demanded load will not be served. Only when a number of conditions are satisfied simultaneously, a network limit will also be a voltage stability limit [B-C].

The above point was raised in discussing the authors' earlier work [D-E]. In their closure they included a simple power system and left its solution to this discusser. Unfortunately, that is not the right system to settle this issue. In the problem formulation of that particular example no explicit load model was used, although a constant MVA and a constant impedance load were depicted on the system diagram. In that example, since the load delivered was identically equal to the generator electrical output, (there being only one generator and one load) the lack of a load model probably went unnoticed by the authors. As such, the limit obtained would be the network limit. Incidentally, since there is only one generator and no (synchronous) reference bus to measure the angle from, the swing equation has no real significance. Also, in that particular formulation, inclusion of a load model (any load model) would introduce a discontinuity in the state variables whenever there is a disturbance that results in a change in the line impedance. Had the authors included one or more additional buses in the system, the lack of a load model would have been obvious. Alternatively, the authors may want to reformulate that problem for a zero power factor (purely reactive) load. The fallacy of the situation would then become apparent.

Note that a constant MVA static load model implies that $\Delta \mathrm{P}=$ $\Delta \mathrm{Q}=0$. It is not a valid load model to address stability issues [C]. It may be used in static analyses after the stability issues have been satisfactorily resolved and provided that the load model does not interfere with proper conclusions.

[A] M.K. Pal, Discussion of "An Investigation of Voltage Instability Problem," by N, Yorino, et al. IEEE Trans. on Power Systems, Vol.7, No.2, pp.600-611, May 1992.

[B] M.K. Pal, "Voltage Stability Conditions Considering Load Characteristics," IEEE Trans. on Power Systems, Vol.7, No.1, pp.243-249, Feb. 1992.

[C] M.K. Pal, "Voltage Stability: Analysis Needs, Modelling Requirement and Modelling Adequacy," IEE Proc. C, Vol.140, No.4, pp.279-286, July 1993.

[D] M.K. Pal, Discussion of reference [6] of the paper, and authors' closure.

[E] M.K. Pal, Discussion of "Point of Collapse Method Applied to AC/DC Power Systems," by C.A. Canizares, F.L. Alvarado, C.L. DeMarco, I. Dobson and W.F. Long, and authors' closure, IEEE Trans. on Power Systems, Vol.7, No.2, pp.673-683, Feb. 1993.

Manuscript received August 27, 1993.

Ian Dobson and Fernando Alvarado: We thank Professor Pai and Dr. Pal for their discussions and reply to their points in order: 


\section{Professor Pai}

1. We expect that tie line powers can be included in the parameter vector $\lambda$ with no difficulty. In general, there is no restriction on the parameters in $\lambda$, but certain parameter choices could lead to a $\Sigma$ whose shape is such that the performance of the algorithm might degrade. There is also more uncertainty in interpreting the meaning of the closest bifurcation to $\Sigma$ if the quantities in the parameter vector are in unrelated units because the closest bifurcation will change if some of the entries in $\lambda$ are scaled differently. Extension of our formulation to consider the case of inter-area transfers is straightforward. One simply substitutes one of the generator equations with an explicit equation to calculate the flow into the desired area, where the total power imported is now unspecified. (Instead of leaving one generator unspecified, one can instead introduce an extra variable for all the area generators.) The procedure is otherwise identical.

2. Our suggestion is that both the worst case load power margin of the paper and the load power margin assuming a predicted pattern of load increase be available to the operator. If either of these voltage collapse indexes falls below their safe threshold values, the screen should alert the operator and provide control options for restoring the index to its safe value. Each control option could specify the amount of control action needed to restore safety if that control was selected. The amount of control action needed is simple to estimate from the first order sensitivity of either index to controls as described in the paper. If there is more than one worst case load power margin of concern or both of the indexes are of concern then control actions effective for all indexes of concern should be recommended. Perhaps the best method for visualizing distance to collapse is entirely graphic. Reference [C1] illustrates various distances to collapse for the 24 bus system. For example, the given-direction normal at collapse can be visualized as shown in the accompanying Figure.

3. The parts of the structural stability limit in parameter space which occur generically are the saddle node bifurcation set $\Sigma$ studied in this paper and the Hopf bifurcation set $\Sigma^{\text {hopf }}$. $\Sigma$ corresponds to voltage collapse (monotonic decrease of system voltages at bifurcation) and $\Sigma^{\text {hopf }}$ corresponds to oscillatory instability (either stable oscillations or oscillatory instability at bifurcation). Some details of the dynamics of the differential equations governing the power system do not affect $\Sigma$ as explained in [12]. However, we think that the dynamics must be fully known to determine $\Sigma^{\text {hopf }}$. This point can be illustrated simply: Suppose we are given 2000 differential equations to describe the power system, and that the first of these differential equations is $\dot{x}_{1}=f_{1}\left(x_{1}, x_{2}, \ldots, x_{2000}, \lambda\right)$. If the dynamics of the first differential equation is changed to $\dot{x}_{1}=2 f_{1}\left(x_{1}, x_{2}, \ldots, x_{2000}, \lambda\right)$, then this change does not affect $\Sigma$ because multiplying the first row of the Jacobian by 2 does not affect the vanishing of its determinant and $\Sigma$ is given by loadings at which the determinant of the Jacobian at the equilibrium vanishes. That is, the zero eigenvalue of the Jacobian is not affected by doubling the first row of the Jacobian. However, nonzero eigenvalues of the Jacobian are usually affected by doubling the first row and, in particular, the imaginary pair eigenvalues signalling a Hopf bifurcation will usually change and the position of $\Sigma^{\text {hopf }}$ will usually be affected. In our opinion, the influence of the unmodelled parts of the power system dynamics on Hopf bifurcation computations needs more careful attention in the literature.

\section{Dr. Pal}

Paragraph 1: We used a specific distribution of generation to match any increase in load when computing the worst case load increase. Since the generators are usually under the control of utilities we assumed that the distribution of the generation increase would be known. However, it is also possible to take into consideration the impact of changes in generation pattern in the computation of load power margins [C2].

Paragraph 2: It is true that if $\left|\lambda-\lambda_{*}\right|$ is small, then a minor perturbation could precipitate voltage collapse. In our approach the load characteristics (including $d y$ namics) are to be specified in the differential equations describing the system. While the load characteristics do affect the system differential equations and its bifurcations, once the load characteristics are agreed upon, the system differential equations are fixed and if those differential equations have $\left|\lambda-\lambda_{*}\right|$ small, then the system is near saddle node bifurcation and instability.

Sensitivity information or eigenvalues can indicate closeness to bifurcation but our opinion is that load power margins are much better. One reason is that eigenvalues and sensitivities vary nonlinearly as the load is linearly increased, making prediction difficult. (The eigenvalues and sensitivities are properties of the linearization of the system and the linearization varies quickly near bifurcation whereas the load power margin takes account of the system nonlinearities.) Another reason is that power systems operation is often thought of in terms of power and it is more straightforward to work directly in terms of load powers.

Paragraph 4: We strongly disagree with Dr Pal's statement that "It has been clearly demonstrated that, in general, saddle node bifurcation has nothing to do with voltage collapse [A]."

First of all, we observe that the following statements are consistent:

(1) Some network loadability limits can be thought of as saddle node bifurcations of static equations.

(2) Voltage collapse is associated with saddle node bifurcation of differential equations.

Dr. Pal seems to use the term saddle node bifurcation exclusively in reference to network limits and then argues that since voltage stability limits often differ from 
network limits, saddle node bifurcations are not related to voltage collapse. In fact, saddle node bifurcations explain and predict voltage stability limits in differential equation models of power systems.

Secondly, a power system model suggested by Dr. $\mathrm{Pal}$ in [C, section 4] has a saddle node bifurcation at which voltage collapse occurs. (This is not surprising or exceptional because nonlinear differential equation models of power systems generically exhibit saddle node bifurcations.) Dr. Pal's power system model consists of simplified equations for a generator, a single lossless line of reactance $j X$ and a purely reactive dynamic load whose reactive admittance $B_{L}$ varies to maintain constant reactive power $Q_{0}$ with a first order lag. We obtained the nonlinear differential equations of Dr. Pal's model by combining from $[\mathrm{C}]$ equations $(4,5,10)$, the equations between (7) and (8) and the equation equating the load current to the load voltage times $B_{L}$ :

$$
\begin{aligned}
& T_{d 0}^{\prime} \frac{d e_{q}^{\prime}}{d t}=e_{f d}-e_{q}^{\prime} \frac{1+B_{L} X_{d}}{1+B_{L} X_{d}^{\prime}} \\
& T_{L} \frac{d B_{L}}{d t}=Q_{0}-\frac{B_{L} e_{q}^{\prime 2}}{\left(1+B_{L} X_{d}^{\prime}\right)^{2}}
\end{aligned}
$$

(We set the reactive compensation $B$ used in [C] to zero for convenience.) The generator quantities are in standard notation and $X_{d}=X+x_{d}, X_{d}^{\prime}=X+x_{d}^{\prime}$. We choose $Q_{0}$ as a quasistatically varying load parameter. The Jacobian is

$$
\left(\begin{array}{cc}
\frac{-\left(1+B_{L} X_{d}\right)}{T_{d 0}^{\prime}\left(1+B_{L} X_{d}^{\prime}\right)} & \frac{-e_{q}^{\prime}\left(x_{d}-x_{d}^{\prime}\right)}{T_{d 0}^{\prime}\left(1+B_{L} X_{d}^{\prime}\right)^{2}} \\
\frac{-2 e_{q}^{\prime} B_{L}}{T_{L}\left(1+B_{L} X_{d}^{\prime}\right)^{2}} & \frac{-e_{q}^{\prime 2}\left(1-B_{L} X_{d}^{\prime}\right)}{T_{L}\left(1+B_{L} X_{d}^{\prime}\right)^{3}}
\end{array}\right)
$$

which agrees with the linearization computed in [C]. The differential equations (DE) have a saddle node bifurcation at $e_{q}^{\prime}=\left(1+X_{d}^{\prime} / X_{d}\right) e_{f d} / 2, B_{L}=1 / X_{d}$ and $Q_{0}=e_{f d}^{2} /\left(4 X_{d}\right)$. The saddle node bifurcation occurs just as the stability condition $B_{L}<1 / X_{d}$ derived in $[C]$ is violated. At bifurcation, the right eigenvector of the Jacobian corresponding to the zero eigenvalue is $v_{*}=\left(-e_{f d}\left(x_{d}-x_{d}^{\prime}\right) / 4,1\right)^{T}$ and dynamic voltage collapse will occur along the center manifold tangent to $v_{*}$ as described in [C3].

Thirdly, Dr. Pal's statement that saddle node bifurcation has nothing to do with voltage collapse is not supported by his discussion [A]. The discussion [A] considers a simple differential/algebraic power system model arising from a dynamic generator swing equation and algebraic equations for a static load model. Dr. Pal discusses singularities of several Jacobians in this particular model, including singularities of the algebraic equations. This discussion does not refer to and is not relevant to the theory of saddle node bifurcations and voltage collapse in differential equation models of power systems. This theory is proposed in [C3] and illustrated with a simple power system with a dynamic load model. We emphasize that the theory is generic and applies to power system models with a variety of dynamic load models. The theory assumes a differential equation model for the power system and quasistatic variation of load parameters (not necessarily powers). Thus the theory addresses small disturbance voltage stability or voltage collapse due to slowly changing parameters. The theory states that the system state will track a stable equilibrium until a saddle node bifurcation is encountered and then the system state will approximately follow the unstable part of the center manifold of the bifurcating equilibrium. This explains why the voltages fall in a voltage collapse. In particular, it applies to Dr. Pal's model as stated above.

Fourthly, contrary to Dr. Pal's apparent impression, the paper assumes dynamic load models so that saddle node bifurcation corresponds to voltage collapse according to the theory of [C3]. The paper states in section 2 that "Underlying differential equation models are assumed but are not necessary for computations [12,14]." Section IV of Reference [12] and the first two paragraphs of section 2 of Reference [14] explain the class of dynamic load models we assume and why we can work with static equations corresponding to the dynamic equations and compute the same answers. Briefly, the dynamics of the load model are a general function of frequency and the real and reactive power balance at the load. We assert that (a) the paper does assume dynamic load models and (b) the computational methods for the closest bifurcation are appropriate to a variety of dynamic load models and (c) once the load dynamics are specified in the system differential equations, the computed saddle node bifurcations will correspond to voltage collapses. Of course the voltage collapse in the model will be more realistic if the dynamic load models can be improved. However, there is not yet consensus on the form and parameters of dynamic load models for voltage collapse studies.

As explained above, load characteristics should be included in the system differential equations because they do influence the voltage collapse bifurcations. We agree that generator reactive power limits can precipitate a voltage collapse as suggested by Dr. Pal in $[\mathrm{C}]$ but, as noted in our paper and Dr. Pal's previous discussion [D], this phenomenon had already been rigorously described in [16] (this first appeared in [19]) and some large system cases computed in [5].

Paragraph 5: Dr. Pal's doubts about saddle node bifurcations, network limits and voltage collapse were also indicated in his previous discussion $[E]$ and were answered in the closure [E] by giving references such as [C3]. The power system example used in the closure [E] did not address these doubts; instead, it addressed Dr. Pal's claim in his discussions $[\mathrm{A}]$ and $[\mathrm{E}]$ that singularity of the power flow Jacobian implied the singularity of the algebraic constraint. Indeed, the power system example 


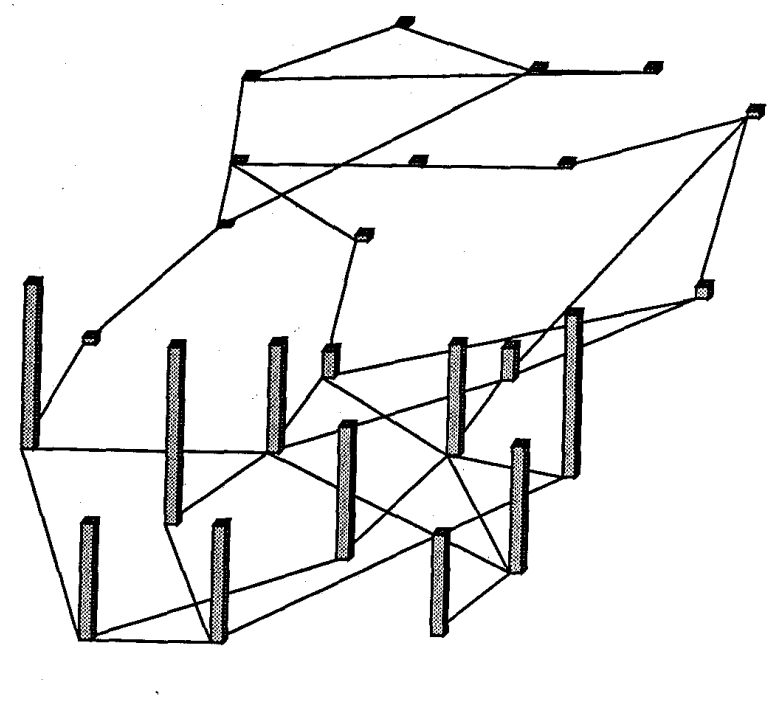

Figure: Spatial visualization of normal vector

in $[\mathrm{E}]$ is a counterexample showing that this claim is false in general. The text for the power system example in [E] specified a constant power load and this load model is adequate for the purpose of disproving Dr. Pal's claim.

We correct the date of reference [E] of Dr. Pal's discussion below.

\section{References}

[C1] F. L. Alvarado, C. Rinzin and R. Adapa, "Visualization of Spatially Differentiated Security Margins," Proceedings of the Power System Computation Conference, August 1993, Avignon, France.

[C2] F. L. Alvarado and Y. Hu, "Dispatch Policy Effects in the Determination of System Security," Proceedings of the NSF Workshop on The Impact of a Less Regulated Environment on Power System Control and Security, Madison, Wisconsin, April 1991, pages 53-77.

[C3] I. Dobson, H.-D. Chiang, Towards a Theory of Voltage Collapse in Electric Power Systems, Systems and Control Letters, Vol. 13, 1989, pp. 253-262.

[E] M.K. Pal, Discussion of "Point of collapse methods applied to AC/DC power systems," by C.A. Canizares, F.L. Alvarado, C.L. DeMarco, I. Dobson and W.F. Long, and authors closure, IEEE Trans. on Power Systems, vol. 7, no. 2, pp. 673-683, May 1992.

Manuscript received October 1, 1993. 